\title{
Homomorphisms of Secret Sharing Schemes: A Tool for Verifiable Signature Sharing
}

\author{
Mike Burmester \\ Information Security Group, Department of Mathematics \\ Royal Holloway - University of London, Egham, Surrey TW20 OEX, U.K. \\ m.burmester@vms.rhbnc.ac.uk.
}

\begin{abstract}
Franklin and Reiter introduced at Eurocrypt '95 verifiable signature sharing, a primitive for a fault tolerant distribution of signature verification. They proposed various praclical protocols. For RSA signatures with exponent $e=3$ and $n$ processors their protocol allows for up to $(n-1) / 5$ faulty processors (in general $(n-1) /(2+e)$ ).

We consider a new unifying approach which uses homomorphisms of secret sharing schemes, and present a verifiable signature sharing scheme for which as many as $(n-1) / 3$ processors can be faulty (for any value of $e$ ), and for which the number of interactions is reduced.
\end{abstract}

\section{Introduction}

Verifiable signature sharing schemes enable the holder of a signed document (who may not be the original signer) to distribute the signature among a set of proxies in such a way that each proxy can verify whether a valid signature can be reconstructed later, even if some of the proxies (but not too many) are malicious or if the original signer is malicious. The signature itself is not revealed until later when it is reconstructed. Verifiable signature sharing is related to the distributed verification of undeniable signatures [18] and to threshold signatures schemes [8]. However there are fundamental differences. With the distributed verification of undeniable signatures, the signed document is public and secret information for the signature is shared. With threshold signatures the power to sign (not to verify) is shared. Such schemes enable any sufficiently large set of shareholders to construct the signature of any document, without any further interaction with the signer.

Verifiable signature sharing has many applications. It can be used to escrow digital cash, by verifiably sharing the signature of a bank for a banknote. Another application is the secure distribution of financial services such as auction bidding [11].

At Eurocrypt '95 Franklin and Reiter presented practical verifiable signature sharing protocols for several signature schemes, including RSA (exponentiation) based signatures with small exponents ${ }^{1} e$ and El Gamal based signatures [10]. In this paper we introduce a new approach based on homomorphisms of secret

\footnotetext{
${ }^{1}$ At the Rump session of Crypto '95 the authors pointed out that their scheme for RSA with $e=3$ was flawed, and that various repairs were considered [12].

U. Maurer (Ed.): Advances in Cryptology - EUROCRYPT '96, LNCS 1070, pp. 96-106, 1996.

(C) Springer-Verlag Berlin Heidelberg 1996
} 
sharing schemes to describe verifiable signature sharing. We use this to extend the Franklin Reiter protocol for El Gamal signatures to a more general setting, and obtain an optimal ${ }^{2}$ verifiable signature sharing scheme. Our construction is natural and simplifies significantly the protocol for RSA based signatures in [10].

\section{Definitions and notation}

Let $A=\{1, \ldots, n\}$ be a set of proxies, $K$ a set of secrets, $S_{1}, \ldots, S_{n}$ sets of shares, and $V=S_{1} \times \cdots \times S_{n}$ the set of $n$-tuples of elements from $S_{i}$.

Definition 1. $[3,19]$ A $(t, n)$-threshold scheme consists of a pair of algorithms, a sharing algorithm $\mathcal{S}$ and a reconstruction algorithm $\mathcal{R} . \mathcal{S}$ is probabilistic and takes as input a secret $k \in K$. It generates an element $c=\left(s_{1}, \ldots, s_{n}\right) \in V$ according to a distribution depending on $k$, and distributes the share $s_{i}$ to proxy $i \in A$. $\mathcal{R}$ is deterministic. It takes as input an element $c \in V$ with up to $n-t$ erasures and will output the secret $k$, if $c$ is an output of $\mathcal{S}$ when $k$ is input. A $(t, n)$-threshold scheme is perfect if $\operatorname{Prob}\left(k \mid s_{i_{1}}, \ldots, s_{i_{t-1}}\right)=\operatorname{Prob}(k)$, for all distributions on $k \in K$, all $\left\{i_{1}, \ldots, i_{t-1}\right\} \subset A$, and all subsets of shares $\left(s_{i_{1}}, \ldots, s_{i_{\ell-1}}\right)$ output by $\mathcal{S}$ on input $k$.

Notation. We write $\mathcal{S}(k)=\left(s_{1}, \ldots, s_{n}\right)$ if (the probabilistic algorithm) $\mathcal{S}$ outputs $\left(s_{1}, \ldots, s_{n}\right)$ on input $k$, and $\mathcal{R}\left(s_{1}, \ldots, s_{n}\right)=k$ if $\mathcal{R}$ outputs $k$ on input $\left(s_{1}, \ldots, s_{n}\right)$. If $B=\left\{i_{1}, \ldots, i_{r}\right\} \subset A$ and $c=\left(s_{1}, \ldots, s_{n}\right) \in V$, then $c_{B}=$ $\left(s_{i_{1}}, \ldots, s_{i_{r}}\right)$. We write $\mathcal{S}_{B}(k)=\left(s_{i_{1}}, \ldots, s_{i_{r}}\right)$. If $r \geq t$ then $\mathcal{R}_{B}\left(s_{i_{1}}, \ldots, s_{i_{r}}\right)=k$. Finally we denote by $\{\mathcal{S}(k)\}$ the set of all $c \in V$ output by $\mathcal{S}$ on input $k$.

We shall assume that the scheme $(\mathcal{S}, \mathcal{R})$ belongs to a family $\left\{\left(\mathcal{S}_{x}, \mathcal{R}_{x}\right)\right\}$ indexed by a parameter $x \in J, J \subset\{0,1\}^{*}$, but for simplicity of notation, when there is no ambiguity, we do not refer explicitly to $x$ and just consider single schemes rather than families. The proxies are polynomially bounded in $|x|$, the binary length of $x$. A threshold scheme is polynomial-time if for any set $B$ of $r \geq t$ proxies, $\mathcal{R}_{B}$ runs in polynomial-time in $r$ and $|x|$. Algorithm $\mathcal{S}$ is not necessarily polynomial-time (in $|x|$ and $n$ ).

Definition 2. A threshold scheme is perfectly zero-knouledge if for any set $B^{\prime}$ of $t-1$ or less proxies, the view of $B^{\prime}$ (i.e. their shares and public information) can be simulated perfectly in expected polynomial-time bounded by $t|x|^{c}, c$ constant. A threshold scheme is perfectly minimal-knowledge if given any $k \in K$ by an oracle, the view of any $r \geq t$ proxies can be simulated perfectly in expected polynomial-time bounded by $r|x|^{c}, c$ constant. For a formal definition the reader is referred to $[15,13,8]$.

Zero-knowledge guarantees that no knowledge at all leaks. Minimal knowledge guarantees that no more than what follows from the joint knowledge of the proxies leaks.

2 The optimality is with respect to the number of faulty processors $T$. In the information theoretic model the bound is $T<n / 3[2,4]$; in the computational model it is $T<n / 2[14]$. 
Remark. A threshold scheme which is perfectly zero-knowledge is also perfect.

Remark. If a $(t, n)$-threshold scheme is perfect and if $\left(s_{i_{1}}, \ldots s_{i_{t-1}}\right)$ are shares of a set $B^{\prime}$ of $t-1$ proxies, then for any $k \in K:\left(s_{i_{1}}, \ldots, s_{i_{t-1}}\right) \in\left\{S_{B^{\prime}}(k)\right\}$.

Definition 3. A $(t, n)$-threshold scheme is an erasure scheme if for any $k \in K$, the shares $\left(s_{i_{1}}, \ldots, s_{i_{t-1}}\right) \in\left\{\mathcal{S}_{B^{\prime}}(k)\right\}$ of any $t-1$ proxies $B^{\prime}$ have a unique extension $\left(s_{1}, \ldots, s_{n}\right) \in\{\mathcal{S}(k)\}$. An erasure scheme is polynomial-time if this extension can be computed in $t$ poly $(|x|)$ time.

Remark. With an erasure scheme, a share-assignment $\left(s_{1}, \ldots, s_{n}\right) \in\{\mathcal{S}(k)\}$ can be reconstructed if $n-t+1$ shares are erased, given $k$.

Definition 4. [5], [8, p. 676] A $(t, n)$-threshold scheme for which the set of secrets is a group $K(*)$ is multiplicative if for any set of $t$ proxies $B=\left\{i_{1}, \ldots, i_{t}\right\}$ there are selection functions $f_{j, B}: S_{j} \rightarrow K, j \in B$, and a public ordering of $\left\{i_{1}, \ldots, i_{t}\right\}$ such that,

$$
k=f_{i_{1}, B}\left(s_{i_{1}}\right) * \cdots * f_{i_{t}, B}\left(s_{i_{t}}\right),
$$

for all $k \in K$ and shares $\left(s_{i_{1}}, \ldots, s_{i_{t}}\right)$ output by $\mathcal{S}$ on input $k$. A multiplicative scheme is polynomial-time if the operation " $*$ " and its inverse are polynomialtime in $|x|$, and if all selection functions are polynomial-time in $|x|$.

Definition 5. Let $\sigma=(\mathcal{S}, \mathcal{R})$ and $\sigma^{\prime}=\left(\mathcal{S}^{\prime}, \mathcal{R}^{\prime}\right)$ be $(t, n)$-threshold schemes with secret sets $K, K^{\prime}$ and share sets $S_{i}, S_{i}^{\prime}, i=1, \ldots, n$, respectively, and let $h_{0}: K \rightarrow K^{\prime}, h_{i}: S_{i} \rightarrow S_{i}^{\prime}, i=1, \ldots, n$, be mappings. The mapping $h=$ $\left(h_{0}, h_{1}, \ldots h_{n}\right)$ is a homomorphism of $\sigma$ to $\sigma^{l}$ if the following conditions hold. For any $k \in K$, if $\mathcal{S}(k)=\left(s_{1}, \ldots, s_{n}\right)$,

- $\left(h_{1}\left(s_{1}\right), \ldots, h_{n}\left(s_{n}\right)\right) \in\left\{\mathcal{S}^{\prime}\left(h_{0}(k)\right)\right\}$

- $h_{0}\left(\mathcal{R}\left(s_{1}, \ldots, s_{n}\right)\right)=\mathcal{R}^{\prime}\left(h_{1}\left(s_{1}\right), \ldots, h_{n}\left(s_{n}\right)\right)$.

That is, the mapping $h$ commutes with the sharing and reconstruction operators.

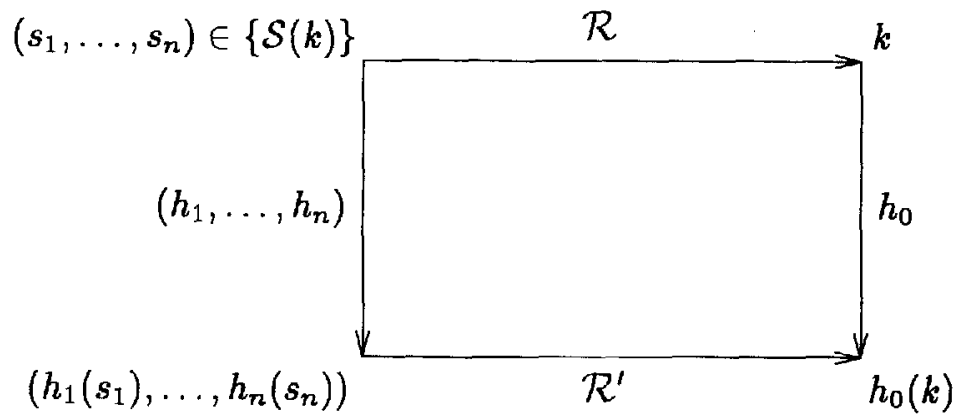

Fig. 1. The commutative diagram of a homomorphism $h: \sigma \rightarrow \sigma^{\prime}$. 
A homomorphism $h$ is polynomial-time if the mappings $h_{i}, i=0,1, \ldots, n$, are polynomial-time in $|x|$.

Notation. If $B=\left\{i_{1}, \ldots, i_{r}\right\}$ then $h_{B}\left(s_{i_{1}}, \ldots, s_{i_{r}}\right)=\left(h_{i_{1}}\left(s_{i_{1}}\right), \ldots, h_{i_{r}}\left(s_{i_{r}}\right)\right)$.

A homomorphism $h: \sigma \rightarrow \sigma^{\prime}$ induces in a natural way a threshold scheme $h(\sigma)=$ $\left(\mathcal{S}^{*}, \mathcal{R}^{*}\right)$ with secret set $K^{*}=h_{0}(K) \subset K^{\prime}$, share sets $S_{i}^{*}=h_{i}\left(S_{i}\right) \subset S^{\prime}$, and $V^{*}=S_{1}^{*} \times \cdots \times S_{n}^{*} \subset V^{\prime} . \mathcal{S}^{*}$ on input $k^{*} \in K^{*}$ outputs $\left(s_{1}^{*}, \ldots, s_{n}^{*}\right) \in V^{*}$ where $s_{1}^{*}=h_{1}\left(s_{1}\right), \ldots, s_{n}^{*}=h_{n}\left(s_{n}\right)$, with the distribution induced by $\left(s_{1}, \ldots, s_{n}\right) \in$ $\{\mathcal{S}(k)\}$ where $h_{0}(k)=k^{*}$. $\mathcal{R}^{*}$ on input $\left(s_{1}^{*}, \ldots, s_{n}^{*}\right) \in V^{*}$ with $s_{1}^{*}=h_{1}\left(s_{1}\right), \ldots$, $s_{n}^{*}=h_{n}\left(s_{n}\right)$ and $k=\mathcal{R}\left(s_{1}, \ldots, s_{n}\right)$, outputs $k^{*}=h_{0}(k)$. We call this, the scheme induced by the homomorhism $h$.

Remark. One should distinguish between homomorphisms of threshold schemes, as introduced here, and homomorphic threshold schemes [1]. With the former no algebraic structure is imposed on the set of secrets or on the shares. However, if "*" is a binary operation on the set of secrets $K$ and "." a binary operation on the sets of shares $S_{i}$, with $\mathcal{R}\left(s_{1}, \ldots, s_{n}\right) * \mathcal{R}\left(s_{1}^{\prime}, \ldots, s_{n}^{\prime}\right)=\mathcal{R}\left(\left(s_{1} \cdot s_{1}^{\prime}\right), \ldots,\left(s_{n} \cdot s_{n}^{\prime}\right)\right)$ for all $\left(s_{1}, \ldots, s_{n}\right),\left(s_{1}^{\prime}, \ldots, s_{n}^{\prime}\right)$ output by $\mathcal{S}$, as in [1], then a homomorphism $h: \sigma \times \sigma \rightarrow \sigma$ is defined in a natural way, with $h_{0}\left(k, k^{\prime}\right)=k * k^{\prime}$ and $h_{i}\left(s_{i}, s_{i}^{\prime}\right)=$ $s_{i} \cdot s_{i}^{\prime}$. So the notion of homomorphisms of sharing schemes is an extension of homomorphic sharing schemes.

Remark. Homomorphisms are an important structure preserving mechanism in algebra. As we shall see they also play an important role in the analysis of cryptographic primitives, by linking verifiable signature sharing to secret sharing.

\section{Verifiable signature sharing based on homomorphisms of secret sharing schemes}

The scheme we describe is based on a homomorphism $h=\left(h_{0}, h_{1}, \ldots, h_{n}\right)$ of the $(t, n)$-threshold schemes $\sigma, \sigma^{\prime}$, and uses the induced threshold scheme $h(\sigma)$. The secrets in $K$ are the signatures $\operatorname{sgn}(m)$ and the elements in $K^{*}=h_{0}(K)$ are the (hashed/processed) messages $m$. We take $\sigma=(\mathcal{S}, \mathcal{R}), h(\sigma)=\left(\mathcal{S}^{*}, \mathcal{R}^{*}\right)$ and $T=t-1$. Our scheme consists of a pair of protocols: a sharing protocol and a reconstruction protocol. In the sharing protocol a dealer $D$ runs $\mathcal{S}$ on input $\operatorname{sgn}(m)$. The dealer gives privately to each of $n$ proxies a share $s_{i}$ of $\operatorname{sgn}(m)$, and sends by reliable broadcast to all proxies the message $M$ and the shares $h_{j}\left(s_{j}\right)$, $j=1, \ldots, T$, in $h(\sigma)$. At the end of this protocol the proxies either accept or reject. In the reconstruction protocol a reconstructor $R$ computes $\operatorname{sgn}(m)$ by majority vote. The scheme is described schematically in Fig 2 . In the upper part we use the zero-knowledge scheme $\sigma=(\mathcal{S}, \mathcal{R})$ and the shares $s_{i}$ are kept secret. In the lower part we use the erasure scheme $h(\sigma)=\left(\mathcal{R}^{*}, \mathcal{S}^{*}\right)$ and the shares $h_{i}\left(s_{i}\right)$ are in the clear. The homomorphism $h$ links the parts. The path $\mathcal{S}(\operatorname{sgn}(m)) \rightarrow \mathcal{S}^{*}(m)$ is used for verification: proxy $i$ checks that the map $h_{i}\left(s_{i}\right)$ of his share $s_{i}$ is the appropriate component of $\left(h_{1}\left(s_{1}\right), \ldots, h_{n}\left(s_{n}\right)\right)$, which he 
gets by extending $\left(h_{1}\left(s_{1}\right), \ldots, h_{T}\left(s_{T}\right)\right)$ using the message $m$. The commutativity of the diagram guarantees correctness.

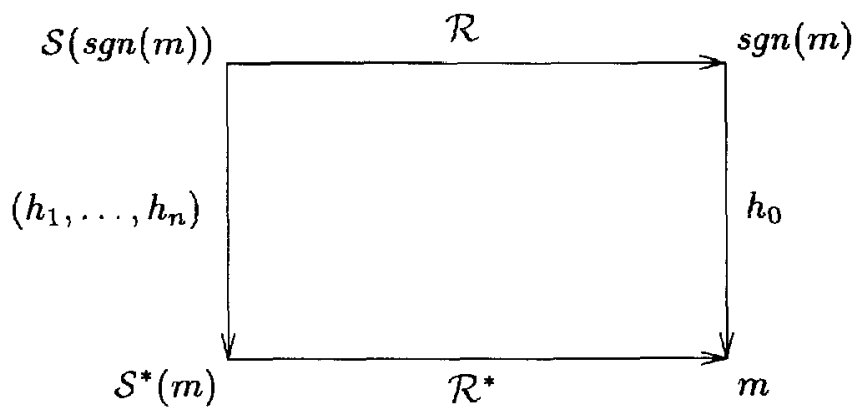

Fig. 2. A diagram for verifiable signature sharing.

We now describe our scheme in detail.

\section{A Verifiable Signature Sharing scheme}

\section{Sharing Protocol}

Step 1 The dealer $D$ uses algorithm $\mathcal{S}$ of $\sigma$ to get shares $s_{1}, \ldots, s_{n}$ of the signature $\operatorname{sgn}(m)$.

a. $D \rightarrow P_{i}$ privately: the share $s_{i}, i=1, \ldots, n$.

b. $D \rightarrow P_{1}, \ldots, P_{n}$ by reliable broadcast: the message $M$, and the shares $h_{1}\left(s_{1}\right), \ldots, h_{T}\left(s_{T}\right)$ in $h(\sigma)$, or something equivalent ${ }^{3}$.

Step 2 Let the broadcast values in Step $1 \mathrm{~b}$ be $M, u_{1}, \ldots, u_{T}$. Each proxy $P_{i}$ computes $m=h a s h(M)$ and makes the following reliable broadcasts to all proxies:

a. COMPLAIN if the share $s_{i} \notin S_{i}$, or if the share $h_{i}\left(s_{i}\right)$ is not the $i$-th component of the extension $\left(u_{1}, \ldots, u_{n}\right) \in\left\{\mathcal{S}^{*}(m)\right\}$ of $\left(u_{1}, \ldots, u_{T}\right)$.

b. ALLOW otherwise.

Step 3 Without further communication, each proxy ACCEPTS if (REJECTS otherwise):

a. At most $T$ proxies COMPLAINed (Nonsensical responses are counted as COMPLAINs).

b. $\left(u_{1}, \ldots, u_{T}\right) \in\left\{\mathcal{S}_{\{1, \ldots, T\}}^{*}(m)\right\}$.

\section{Reconstruction Protocol}

Step 1 Each proxy $P_{i}$ sends to the reconstructor $R$ the following information:

a. The shares $s_{i}$ sent in Step 1a of the Sharing Protocol.

b. The values $M, u_{1}, \ldots, u_{T}$ broadcast in Step $1 \mathrm{~b}$ of the Sharing Protocol.

\footnotetext{
${ }^{3}$ For example a polynomial-time invertible bijection of these, as in [10].
} 
Step 2 Without further communication:

a. The reconstructor $R$ finds $\bar{M}, \bar{u}_{1}, \ldots, \bar{u}_{T}$ by majority vote on the lists received in Step $1 \mathrm{~b}$ (of this protocol).

b. $R$ discards every share $s_{i}$ received in Step 1 a (of this protocol) that is inconsistent with $\bar{M}, \bar{u}_{1}, \ldots, \bar{u}_{T}$, in $h(\sigma)$, i.e., for which the share $s_{i} \notin S_{i}$, or the share $h_{i}\left(s_{i}\right)$ is not the $i$-th component of the extension $\left(\bar{u}_{1}, \ldots, \bar{u}_{n}\right) \in\left\{\mathcal{S}^{*}(\bar{m})\right\}$ of $\left(\bar{u}_{1}, \ldots, \bar{u}_{T}\right)$.

c. Let $B=\left\{i_{1}, \ldots, i_{T}\right\}$ be the set of proxies with the remaining shares. $R$ computes $\mathcal{R}_{B}\left(s_{i_{1}}, \ldots, s_{i_{r}}\right)$ and outputs this as its signature.

We shall prove that this protocol achieves a slightly stronger goal than in [10].

Definition 6. A verifiable signature sharing scheme is $T$-resilient if the following conditions hold.

(i) (Completeness) If the dealer is honest and at most $T$ proxies are faulty then honest proxies accept, and the reconstruction is successful.

(ii) (Soundness) If at most $T$ proxies are faulty and an honest proxy accepts, then every proxy accepts and the reconstruction is successful.

(iii) (Secrecy) Anything that can be computed by an adversary who, (a) controls up to $T$ faulty proxies who have participated in earlier sharing protocols with an honest dealer and (b) has access to the view of the reconstructor in earlier reconstructions, can also be computed by the adversary without participating in any of these protocols.

Remark. Secrecy is based on the simulation of the view of the adversary. To simulate the shares of faulty (dishonest) proxies in the sharing protocols, $\sigma$ must be zero-knowledge. To simulate the (uncorrupted) shares of the honest proxies revealed in the reconstruction protocols, $h(\sigma)$ must be minimal knowledge.

Remark. Our definition of secrecy is stronger than that in [10], since we allow the adversary to access earlier reconstruction protocols. We feel that this is an essential security requirement for verifiable signature sharing. Although the schemes proposed in [10] satisfy the stronger secrecy requirement, their model overlooked this aspect.

Remark. If $h_{0}$ is a one-way function then this scheme can also be used as a verifiable secret sharing scheme with heuristic security. The security is only heuristic because with one-way functions there is no guarantee that the input will not leak.

Theorem 7. Suppose that $\sigma, \sigma^{\prime}$ are polynomial-time $(t, n)$-threshold schemes with $t=T+1$ and $T \leq(n-1) / 3$. The protocol above is a $T$-resilient verifiable signature sharing scheme if $h: \sigma \rightarrow \sigma^{\prime}$ is a polynomial-time homomorphism and if the following conditions hold:

1. All the mappings $h_{i}, i=0,1, \ldots, n$, are injections.

2. $\sigma$ is a perfectly zero-knowledge polynomial-time erasure scheme, and membership in $S_{i}$ for all $i$ can be checked in polynomial-time. 
3. $\sigma^{\prime}$ is a polynomial-time erasure scheme, and membership in $\left\{\mathcal{S}_{\{1, \ldots, T\}}^{*}(m)\right\}$ can be checked in polynomial-time.

To prove this theorem we need the following results.

Lemma 8. Let $\sigma, \sigma^{\prime}$ be polynomial-time threshold schemes with $\sigma$ perfectly zeroknowledge, $\sigma^{\prime}$ a polynomial-time erasure scheme, and $h: \sigma \rightarrow \sigma^{\prime}$ a polynomialtime homomorphism. Then $h(\sigma)$ is perfectly minimal-knowledge.

Proof. Since $h$ is a homomorphism, $h(\sigma)$ is a perfectly zero-knowledge erasure scheme (it is not necessary that $h(\sigma)=\sigma^{\prime}$ ). We describe an expected polynomialtime simulator $M^{*}$ which given any $k^{*} \in K^{*}$ simulates shares of any set of $t$ proxies $B=\left\{i_{1}, \ldots, i_{t-1}, i_{t}\right\}$ in $h(\sigma)$. Now $\sigma$ is perfectly zero-knowledge, so there is a simulator $M$ which simulates the shares in $\sigma$ of the set of $t-1$ proxies $B^{\prime}=\left\{i_{1}, \ldots, i_{t-1}\right\}$. To simulate the shares of $B$ in $h(\sigma), M^{*}$ first simulates the shares of $B^{\prime}$ using $M$, to get $\left(s_{i_{1}}, \ldots, s_{i_{t-1}}\right)$ with a distribution identical to the real one. Then $M^{*}$ computes $\left(h_{i_{1}}\left(s_{i_{1}}\right), \ldots, h_{i_{t-1}}\left(s_{i_{t-1}}\right)\right)$. Because $h(\sigma)$ is an erasure scheme, given any $k^{*} \in K^{*}, M^{*}$ can compute its unique extension $c_{B}^{*} \in\left\{\mathcal{S}_{B}^{*}\left(k^{*}\right)\right\}$ (perfect zero-knowledge guarantees that $c_{B^{\prime}}^{*}$ belongs to $\left\{\mathcal{S}_{B^{\prime}}^{*}\left(k^{*}\right)\right\}$ - cf. the Remarks after Definition 2). Finally $M^{*}$ outputs $c_{B}^{*}$ as the simulated shares for $B$.

Corollary 9. If the conditions in the Lemma are satisfied and $h_{0}$ is a one-way function then each $h_{i}, i=1, \ldots, n$, is one-way.

Proof. Suppose that it is easy to invert $h_{j}$. Let $B=\left\{i_{1}, \ldots, i_{t}\right\}$ be a set of $t$ distinct proxies with $j=i_{t}$ and let $B^{\prime}=\left\{i_{1}, \ldots, i_{t-1}\right\}$. We describe a polynomialtime algorithm $\mathcal{A}$ which inverts $h_{0}$, that is, given $k^{*} \in K^{*}$ computes a $k \in K$ with $h_{0}(k)=k^{*}$. As in Lemma $8, \mathcal{A}$ chooses shares $s_{i_{1}}, \ldots, s_{i_{t-1}}$ for $B^{\prime}$ and computes $h_{i_{1}}\left(s_{i_{1}}\right), \ldots, h_{i_{t-1}}\left(s_{i_{t-1}}\right)$ and their unique extension $c^{*}$. Then $\mathcal{A}$ inverts the $j$-th component $s_{j}^{*} \in h_{j}\left(S_{j}\right)$ of $c^{*}$ to get an $s_{j} \in S_{j}$ such that $h_{j}\left(s_{j}\right)=s_{j}^{*}$, and computes $k=\mathcal{R}_{B}\left(s_{i_{1}}, \ldots, s_{i_{t-1}}, s_{j}\right)$. Since $h$ is a homomorphism, $h_{0}(k)=k^{*}$.

Corollary 10. A polynomial-time threshold scheme which is a perfectly zeroknowledge polynomial-time erasure scheme is perfectly minimal knowledge.

Proof. Take $\sigma=\sigma^{\prime}$ and $h$ the identity homomorphism in Lemma 8.

\section{Proof of the theorem:}

Completeness: This follows directly from the fact that $h$ is a homomorphism.

Soundness: If one honest proxy accepts then all honest proxies accept. We must show that in this case the reconstruction is successful. First observe that $h(\sigma)$ is a polynomial-time erasure scheme. So $\left(u_{1}, \ldots, u_{T}\right)$ has a unique extension $c^{*}=\left(s_{1}^{*}, \ldots, s_{n}^{*}\right)$ in $h(\sigma)$ with $\mathcal{R}\left(c^{*}\right)=m$, which can be computed in polynomialtime. Allowing for up to $T$ COMPLAINs and up to $T$ faulty proxies we see that at most $2 T$ of the components of $c^{*}$ are in error in the majority vote count. Since $n-2 T \geq T+1=t$, there is a set $B=\left\{i_{1}, \ldots, i_{r}\right\}$ of $r \geq t$ proxies with shares $\left(h_{i_{1}}\left(s_{i_{1}}\right), \ldots, h_{i_{n}}\left(s_{i_{n}}\right)\right) \in\left\{\mathcal{S}_{B}^{*}(m)\right\}$. We are assuming that the mappings $h_{i}$ are injections. It follows that $\left(s_{i_{1}}, \ldots, s_{i_{n}}\right) \in\left\{\mathcal{S}_{B}(k)\right\}$. Because $h$ is a homomorphism and $h_{0}$ is injective, $k=\operatorname{sgn}(m)$. 
Secrecy: We have to simulate, essentially, tuples $\left(h_{\{1, \ldots, T\}}\left(c_{\{1, \ldots, T\}}\right), c_{B^{\prime}}\right)$, with $\left|B^{\prime}\right| \leq T$, for the sharing protocol, and tuples $(h(c), c, \operatorname{sgn}(m))$ with up to $T$ corrupted entries in $c$ for the reconstruction protocol. Since the sharing algorithm $\mathcal{S}$ distributes shares independently for each run of the verifiable signature sharing scheme (the dealer is honest), it is sufficient to simulate one run. Furthermore we only need to simulate the uncorrupted parts. Now $\sigma$ is zero-knowledge, so $c_{B^{\prime}}$ can be simulated. Therefore given the unsigned (hashed/processed) message $m$, $\left(h_{\{1, \ldots, T\}}\left(c_{\{1, \ldots, T\}}\right), c_{B^{\prime}}\right)$ can be simulated, because $h(\sigma)$ is a polynomial-time erasure scheme. For the reconstruction protocol we have to simulate the uncorrupted parts of $(h(c), c, s g n(m))$. In this case the simulator is also given the signature $\operatorname{sgn}(m)$. By Corollary $10, \sigma$ is minimal-knowledge, so $c$ can be simulated. Then $h(c)$ can be simulated, since $h$ is polynomial-time.

Remark. For this scheme $T \leq(n-1) / 3$. The upper (exclusive) bound for the number faulty processors in a fault tolerant distributed computation in the information theoretic model is $n / 3[2,4]$. So the scheme is optimal in this respect.

\section{Applications}

\subsection{A Verifiable Signature Sharing scheme based on the Desmedt- Frankel zero-knowledge RSA threshold scheme}

Take $\sigma$ to be the zero-knowledge RSA threshold scheme in [8] with $\sigma^{\prime}=\sigma$. This scheme is homomorphic (cf. the Remark after Definition 5) and uses polynomial interpolation for the reconstruction of the secret. In this case however the coefficients of the polynomials are not taken from a field but from a ring extension $Z[u]$ of the integers. We briefly explain this scheme.

Let $N$ be a composite number, $n$ be polynomially bounded in $|N|, q$ a prime with $q \geq n+1$, and $Z[u]=Z[x] /\left(\left(x^{q}-1\right) /(x-1)\right)$ be the ring of all polynomial expressions in $u$ with integer coefficients taken modulo the cyclotomic polynomial $\left(u^{q}-1\right) /(u-1)$. The set of secrets $K$ and the share sets $S_{i}, i=1, \ldots, n$, have a group structure. $K$ is the multiplicative group $Z_{N}^{*}(\cdot)$ and $S_{i}$ is the $Z[u]$-module $M=Z[u] \otimes_{Z} Z_{N}^{*}$, the tensor product [17] of $Z[u](+)$ and $Z_{N}^{*}(\cdot)$. It is easy to see that $M \cong\left(Z_{N}^{*}\right)^{q-1}=Z_{N}^{*} \times \cdots \times Z_{N}^{*}$. Each shareholder is given a share $s_{i} \in S_{i}$, and a set of $r \geq t$ shareholders $B=\left\{i_{1}, \ldots, i_{\tau}\right\}$ reconstructs the secret by using

$$
\mathcal{R}_{B}\left(s_{i_{1}}, \ldots, s_{i_{r}}\right)=\operatorname{proj}_{1}\left(\sum_{i \in B} y_{i_{i} B} \cdot s_{i}\right)
$$

where proj $_{1}$ is the projection of the elements of $\left(Z_{N}^{*}\right)^{q-1}$ onto their first coordinate in $Z_{N}^{*}$, the sum is the internal module operation, the product is the external module operation, and the $y_{i, B}$ are the Lagrange interpolation coefficients,

$$
y_{i, B}=\prod_{j \in B, j \neq i}\left(0-u^{j}\right) /\left(u^{i}-u^{j}\right),
$$

which are invertible elements in $Z[u][8]$. 
For our application, $h_{0}: Z_{N}^{*} \rightarrow Z_{N}^{*}: x \rightarrow x^{e} \bmod N$, where $\operatorname{gcd}(e, \phi(N))=1$, and $h_{i}:\left(Z_{N}^{*}\right)^{q-1} \rightarrow\left(Z_{N}^{*}\right)^{q-1}:\left(x_{1}, \ldots, x_{q-1}\right) \rightarrow\left(x_{1}^{e}, \ldots, x_{q-1}^{e}\right), i=1, \ldots, n$. Clearly $h$ is injective. Also $h_{i}(s)=e \cdot s, s \in\left(Z_{N}^{*}\right)^{q-1}$, using the external module operation. Furthermore the mappings $h_{i}$ commute with proj $_{1}$ and the module operations (i.e. $h$ is a morphism), since

$$
\begin{aligned}
\operatorname{proj}_{1}\left(h_{i}\left(s_{i}\right)\right) & =\operatorname{proj}_{1}\left(e \cdot s_{i}\right)=h_{0}\left(\operatorname{proj}_{1}\left(s_{i}\right)\right), \text { and } \\
\sum_{i} y_{i} \cdot h_{i}\left(s_{i}\right) & =\sum_{i} y_{i} \cdot\left(e \cdot s_{i}\right)=e \cdot\left(\sum_{i} y_{i} \cdot s_{i}\right)=h_{j}\left(\sum_{i} y_{i} \cdot s_{i}\right),
\end{aligned}
$$

for all $s_{i} \in\left(Z_{N}^{*}\right)^{q-1}, y_{i} \in Z[u]$. It follows that conditions (2) in Section 2 hold, and that $h$ is a homomorphism of $\sigma$ onto $\sigma$. It is easy to prove that all the other conditions of Theorem 7 are satisfied. In particular $\sigma$ is an erasure scheme. Also $\left\{\mathcal{S}_{\{1, \ldots, T\}}(k)\right\}=\left(Z_{N}^{*}\right)^{T}$, so that one only has to check membership in each of the components $Z_{N}^{*}$, to check membership in $\left\{\mathcal{S}_{\{1, \ldots, T\}}(k)\right\}$. Thus we have a $T$-resilient verifiable signature sharing scheme.

\subsection{A Verifiable Signature Sharing scheme based on the Desmedt- DiCrescenzo-Burmester zero-knowledge threshold scheme}

Take $\sigma$ to be the perfect zero-knowledge threshold scheme in [5] with $\sigma^{\prime}=\sigma$. This scheme is multiplicative and the set of secrets $K(*)$ can be any group (not necessarily abelian). The shares are sets of sub-shares which are tuples (list $t_{a}, k_{a}$ ). A set $B$ of $t$ distinct proxies can recompute the secret $k$ by taking the product of appropriate $k_{a}$ 's whose selection depends on $B$, and is determined by the lists list $a$. The lists define selection functions $f_{j, B}\left(s_{j}\right), j \in B$, which are such that equation (1) in Section 2 holds. The functions $f_{j, B}$ commute with any homomorphism of the multiplicative group $K(*)$. We take $h_{0}$ to be a one-way injective group homomorphism of a polynomial-time group $K(*)$ (for which the operation " $*$ " and its inverse are polynomial-time). As in the previous case we use $h_{0}$ to define injections $h_{i}$ on the shares $s_{i}$ (for which each (list ${ }_{a}, k_{a}$ ) is mapped to (list $\left.{ }_{a}, h_{0}\left(k_{a}\right)\right)$ ). Then conditions (2) hold, as can easily be checked, and we get a homomorphism from $\sigma$ to $\sigma$. It is easy to check that $\sigma$ is an erasure scheme when $t=2$. All conditions of Theorem 7 are then satisfied. So we get a 1 -resilient verifiable signature scheme. In this case the size of the shares is reduced, roughly, from $n \log N$ to $(\log n)(\log N)$.

\section{Conclusion}

We have shown that by using homomorphisms of secret sharing schemes we can get RSA based verifiable signature sharing schemes which are optimal with respect to the number of faulty processors. Our approach is natural, uses existing secret sharing schemes, and simplifies significantly the RSA based protocol of Franklin and Reiter [10]. 


\section{Acknowledgements}

The author discussed the topic of this paper with Yvo Desmedt during Eurocrypt ' 95 , who independently made the observation that a generalization of Pedersen's undeniable signatures could be used with the Desmedt-Frankel zeroknowledge threshold scheme to improve the Franklin-Reiter scheme.

The author wishes to thank Yvo Desmedt, Simon Blackburn and Peter Wild for many useful discussions.

\section{References}

1. J.C. Benaloh: Secret sharing homomorphisms: Keeping shares of a secret secret. In: A. Odlyzko (ed.): Advances in Cryptology, Proc. of Crypto '86. Lecture Notes in Computer Science 263, Berlin: Springer 1987, pp. 251-260

2. M. Ben-Or, S. Goldwasser, A. Wigderson: Completeness theorems for noncryptographic fault-tolerant distributed computation. Proceedings of the twentieth annual ACM Symp. Theory of Computing, STOC, 1988, pp. 1-10

3. G. R. Blakley: Safeguarding cryptographic keys. In: Proc. Nat. Computer Conf. AFIPS Conf. Proc., 48, 1979, pp. 313-317

4. D. Chaum, C. Crépeau and I. Damgård: Multiparty unconditionally secure protocols. Proceedings of the twentieth annual ACM Symp. Theory of Computing, STOC, 1988, pp. 11-19

5. Y. Desmedt, G. Di Crescenzo, and M. Burmester: Multiplicative non-abelian sharing schemes and their application to threshold cryptography. In: J. Pieprzyk, R. Safavi-Naini (eds.): Advances in Cryptology - Asiacrypt '94. Lecture Notes in Computer Science 917. Berlin: Springer 1995, pp. 21-32

6. A. De Santis, Y. Desmedt, Y. Frankel, and M. Yung: How to share a function securely. In: Proceedings of the twenty-sixth annual ACM Symp. Theory of Computing (STOC), 1994, pp. 522-533. Full paper in preparation.

7. Y. G. Desmedt: Threshold cryptography. European Trans. on Telecommunications, 5(4), pp. 449-457 (1994)

8. Y.G. Desmedt, Y. Frankel: Homomorphic zero-knowledge threshold schemes over any finite abelian group. SIAM Journal on Discrete Mathematics, 7(4), 667-679, (1994)

9. M. De Soete, J-J. Quisquater, and K. Vedder: A signature with shared verification scheme. In: G. Brassard (ed.): Advances in Cryptology - Crypto '89. Lecture Notes in Computer Science 435. Berlin: Springer 1990, pp. 253-262

10. M. K. Franklin and M. K. Reiter: Verifiable signature sharing. In: L.C. Guillou, J.J. Quisquater (eds.): Advances in Cryptology - Eurocrypt '95. Lecture Notes in Computer Science 921. Berlin: Springer 1995, pp. 50-63

11. M. K. Franklin and M. K. Reiter: The design and implementation of a secure auction service. IEEE Symposium on Security and Privacy. Oakland CA, 1995

12. M. K. Franklin and M. K. Reiter: A linear protocol failure for RSA with exponent three. Presented at the Rump session of Crypto '95, Santa Barbara, California, USA, August 27-31, 1995.

13. Z. Galil, S. Haber, and M. Yung: Minimum-knowledge interactive proofs for decision problems. Siam J. Comput., 18(4), 711-739 (1989) 
14. O. Goldreich, S. Micali and A. Wigderson: How to play any mental game. Proceedings of the Nineteenth annual ACM Symp. Theory of Computing, STOC, 1987, pp. 218-229

15. S. Goldwasser, S. Micali, and C. Rackoff: The knowledge complexity of interactive proof systems. Siam J. Comput., 18(1), 186-208 (1989)

16. L. Harn: Digital signature with $(t, n)$ shared verification based on discrete logarithms. Electronics Letters, 29(24), 2094-2095 (1993)

17. N. Jacobson: Basic Algebra I. W. H. Freeman and Company, New York (1985)

18. T. P. Pedersen: Distributed provers with applications to undeniable signatures. In: D.W. Davies (ed.): Advances in Cryptology - Eurocrypt '91. Lecture Notes in Computer Science 547, Berlin: Springer 1991, pp. 221-242

19. A. Shamir: How to share a secret. Commun. ACM, 22, 612-613 (1979) 